Relations industrielles

Industrial Relations

\title{
Robert D. BUREAU, Pierre MACKAY : Le droit dans tous ses états. Montréal, Wilson \& Lafleur, 1987, 620 p., ISBN 2-89127-07602
}

\section{Fernand Morin}

Volume 45, numéro 3, 1990

URI : https://id.erudit.org/iderudit/050622ar

DOI : https://doi.org/10.7202/050622ar

Aller au sommaire du numéro

Éditeur(s)

Département des relations industrielles de l'Université Laval

ISSN

0034-379X (imprimé)

1703-8138 (numérique)

Découvrir la revue

Citer ce compte rendu

Morin, F. (1990). Compte rendu de [Robert D. BUREAU, Pierre MACKAY : Le droit dans tous ses états. Montréal, Wilson \& Lafleur, 1987, 620 p., ISBN 2-89127-07602]. Relations industrielles / Industrial Relations, 45(3), 640-643. https://doi.org/10.7202/050622ar

Tous droits réservés (C Département des relations industrielles de l'Université Laval, 1990
Ce document est protégé par la loi sur le droit d'auteur. L’utilisation des services d’Érudit (y compris la reproduction) est assujettie à sa politique d'utilisation que vous pouvez consulter en ligne.

https://apropos.erudit.org/fr/usagers/politique-dutilisation/ 
La conclusion de l'ouvrage se présente, à toutes fins utiles, comme une réflexion sur le droit de refus en tant que nouveau mode de régulation des risques de travail, réflexion qui se termine par cette note: «Bref, si la reconnaissance du droit de refus dans la Loi sur la santé et la sécurité du travail a provoqué une révolution, ce fut une révolution tranquille» (p. 315). Pour contrer la sous-utilisation du droit dans le secteur non syndiqué, les auteurs suggèrent de rappeler l'idée du «représentant multi-établissements à la prévention». Heureuse recommandation! Car il convient de s'interroger sur le fait que seulement $2 \%$ des refus proviennent de travailleurs non syndiqués alors qu'ils constituent $64,2 \%$ de la population active (1983).

En définitive Le droit de refus: une révolution tranquille est un ouvrage bien fait et agréable à lire malgré son contenu souvent très technique. On doit le considérer comme un outil de référence essentiel à toute personne oeuvrant en matière de santé et de sécurité au travail. Mais avant toute chose il rendra de nombreux services dans les milieux de la recherche et de l'enseignement.

Rodrigue BLOUIN

Université Laval

Le droit dans tous ses états, sous la direction de Robert D. Bureau et Pierre Mackay, Montréal, Wilson et Lafleur Ltée, 1987, 620 pages, ISBN 2-89127-076-2

Sous ce titre accrocheur, trente professeurs du département des sciences juridiques de l'Université du Québec à Montréal publient ensemble autant d'articles regroupés sous ces cinq rubriques: l'État, les personnes, les conditions de vie, les organisations et l'émergence d'une science juridique. Il appert que cet ouvrage coöncide avec le quinzième anniversaire du département des sciences juridiques, aussi les auteurs ont-ils dédié ce collectif à leurs étudiants. Pour des raisons non exprimées, les co-directeurs ne profitèrent pas de l'occasion pour faire le point de ces quinze années d'expérience tant scientifique que pédagogique. Outre cette première discrétion, ils ont également préféré un préfacier d'une autre université que la leur soit Monsieur le doyen Roderick A. MacDonald de l'Université McGill.

Il est possible que le lecteur ne soit pas intéressé par chacun de ces trente articles puisque l'on y traite également du droit constitutionnel, du droit pénal, du droit administratif, du droit civil et du droit du travail. Par ailleurs, la lecture de l'ensemble de l'ouvrage permettrait de saisir le droit québécois d'une manière quelque peu différente des traités traditionnels. Ce n'est ni le style, ni même les méthodes employées qui confèrent à cet ouvrage sa spécificité, mais bien le point d'appui généralement retenu par les auteurs soit une analyse politique de la production législative de ces quinze ans.

Les seuls titres des sept articles formant le chapitre $1 \mathbf{L}$ '́tat évoquent bien le caractère de l'oeuvre: la crise de l'État, le déclin de l'empire britannique, le danger qui croît avec l'usage, du soliloque à la polysémie, etc. Peut-être ces titres-réclames sauront-ils retenir l'attention de certains lecteurs qui autrement ne s'y arrêteraient pas! On remarque cependant que plusieurs auteurs embrassent trop grand et en conséquence, passent rapidement d'une question à une autre. Il s'en suit que l'analyse retarde. Parmi ces sept articles, nous soulignons le texte de Dorval Brunelle qui tisse un lien entre les rapports des «sages» commandés par le gouvernement libéral du Québec et la Loi constitutionnelle de 1982. S'ajoute la note de Georges A. Lebel qui souligne l'importance de considérer les libertés garanties aux chartes comme un tout dans le cadre et pour les fins d'une société politique et non comme de simples encouragements à l'égocentrisme: «Toute liberté qui n'est pas solidarité ne peut être que privilège et exploitation» (p. 115). 
Le chapitre 2, Les personnes, comprend six articles où on traite de la famille (Renée Joyal), de l'union stable hors mariage (Serge Allard), de la sexualité et du droit pénal (Johanne Doucet et Lucie Lemonde), de l'égalité de la femme en emploi (Lucie Lamarche), des jeunes (Robert D. Bureau) et finalement, des réfugiés (Daniel Paquin). Par les thèmes traités et les nombreux rappels de l'évolution des lois promulguées sous ces différents chefs depuis quinze ans, ce chapitre illustre d'une façon éclatante les mutations rapides que connut la société québécoise. Ces auteurs relatent le traitement juridique retenu au sujet de questions aussi difficiles que celles de l'homosexualité, la prostitution, la pornographie, etc. On rappelle de façon détaillée la difficile sinon pénible et tortueuse évolution des règles devant assurer l'égalité des femmes en matière d'emploi (Lucie Lamarche). Cette démonstration permet de constater que Québec ne constitue nullement un laboratoire-pilote dynamique et imaginatif en ces matières. Finalement, nous soulignons l'exposé de Daniel Paquin portant sur les réfugiés. Peu d'auteurs développent cette question au Québec et pourtant, le traitement que l'on réserve aux «nouveaux arrivants» traduit assez fidèlement le degré de civilisation d'une société.

Au chapitre 3, Les conditions de vie, on y traite d'abord de l'évolution des régimes de compensation pour préjudices corporels (C.A. Ducharme et Claude Masse). Cinq articles suivant portant respectivement sur le logement (Claude Thomasset), les droits des usagers du système de santé (Jean-Pierre Ménard), l'insécurité du revenu des accidentés du travail (Katherine Lippel), la sécurité du revenu (Yves Bélanger) et le droit de la consommation (Claude Masse et Pauline Roy). Nous soulignons l'article sur le droit et l'environnement de Maryse Grandbois bâtit sur la prémisse que l'État se sert du droit pour aménager de nouveaux rapports spaciaux ce qui remettrait en cause le jus utendi et abutendi du propriétaire. Semblable au droit du travail, le droit de l'environnement, rappelle-t-on, se construit par à-coup, au gré des catastrophes et en réponse aux pressions collectives.

Le chapitre 4, Les organisations retint davantage notre attention compte tenu du créneau de cette revue. Carole Jobin fait l'historique de l'évolution des organes administratifs du Code du travail, c'est-à-dire de la première commission des relations ouvrières à l'éventuelle nouvelle commission et traite des facteurs qui expliqueraient la judiciarisation des rapports collectifs du travail au Québec. Outre son style alerte et dynamique, nous aurions aimé que l'auteur étudie davantage l'actuel système administratif retenu depuis 1961 et discute des motifs invoqués pour l'écarter par une loi sanctionnée depuis 1987 sans que l'on ose encore la promulguer. Les enseignements du Conseil canadien des relations du travail que l'on nous transmet ne nous ont guère convaincu de la pertinence de telles importations. Jean-Serge Masse fait rapport d'une étude détaillée relative au devoir d'une juste représentation imposé au syndicat. Outre l'analyse descriptive des données recueillies, cet auteur soulève d'innombrables «lieux d'interrogation» sans nous fournir autant d'éléments de réponses de son cru.

Nous retrouvons sous la plume de Jacques Desmarais un article intéressant relatif à l'expérience québécoise de la négociation collective dans les secteurs publics. Ce rappel des péripéties que le Québec connut et où se confondent, dans l'ordre et le désordre, loi, négociation, décret et loi spéciale est certes utile, notamment pour fins pédagogiques. En raison de l'expérience de l'auteur en ce domaine, nous regrettons cependant de ne pouvoir y lire son analyse de la délicate question à savoir si un tel régime de relations du travail ne serait pas inexorablement voué à l'échec et que, de part et d'autre, on n'oserait soulever. L'article se termine par la suggestion d'un programme d'étude et de recherche sans proposer quelques pistes adaptées au secteur public. En somme, ne devrait-on pas discuter s'il est encore possible de réduire l'État au seul statut d'employeur et, par voie de conséquence, d'assimiler un fonctionnaire à un commis de magasin? L'auteur nous propose plutôt un grand débat philosophico-juridique de la fonction du droit dans la société. Ce quatrième chapitre se termine par deux brefs articles portant sur la 
société anonyme avec ou sans but lucratif. Les auteurs Georges A. Lebel pour un et Paul Martel pour l'autre font voir que la législation québécoise en ce domaine s'inspire ou imite les lois fédérales ou ontariennes tout en conservant un certain retard.

Finalement, le chapitre 5, L'émergence d'une science juridique se situe dans le créneau principal retenu par ce département des sciences juridiques du moins en sa phase inaugurale. Jean-Marie Fecteau souligne que «[...] Le droit est non seulement un champ d'étude, une dimension constitutive de l'existence sociale, mais aussi une pratique» (p. 507) d'où la nécessité d'en faire une étude historique en tenant compte de toutes ces facettes. On y fait rapidement l'histoire de l'enseignement de l'histoire du droit et de la spécificité de ce champ d'étude et on parle même de son autonomie (p. 508). Ce dernier qualificatif nous paraîtrait quelque peu abusif si l'auteur ne reconnaissait pas d'emblée que l'isolement du droit y compris son histoire ne permettrait nullement de saisir sa totale réalité (p. 512). René Laperrière se met à la recherche de la science juridique et discute des postulats et de la méthodologie retenus par le Groupe consultatif sur la recherche et les études de droit commandés par le Conseil de recherche en sciences humaines du Canada. Selon lui, cette dernière étude de 1982 nous conduirait «[...] au seuil d'une recherche théorique approfondie sur le droit et la profession juridique, dans leurs rapports avec la politique et la société. L'ambition des auteurs s'arrête là, où il nous invite à prendre le relai» (p. 520). Aussi est-il intéressant de savoir justement comment ce département des sciences juridiques sut prendre ce relai au cours de ces dernières huit années. Comme le rappelle l'auteur, cette mission devait être celle des professeurs d'universités qui «[...] jouissent d'une sécurité d'emploi qui est justement censée leur permettre d'exercer en toute liberté académique ce sens critique qui fait si cruellement défaut au milieu juridique» (p. 523). L'auteur tente par la suite d'expliquer l'absence d'études critiques du droit et de véritables juristes chercheurs pour assumer les risques inhérents à leur conclusion parce que, dit-il, on étudierait le droit pour se sécuriser: «[...] la motivation principale consiste à vouloir connaître le droit pour se sécuriser personnellement par rapport à ce domaine perçu par la majorité comme étant essentiellement répressif, ou comme un moyen de tromper la bonne foi des gens»; (p. 523). Peu après cette surprenante tirade, l'auteur apprécie ainsi les études des juristes: «Les juristes ont mis au point des méthodes très raffinées et très rigoureuses d'analyse des textes juridiques (lois, règlements, décisions judiciaires), ce qui leur a fait vouer un certain culte à l'écrit» (p. 525). Selon cet auteur, cet article comprendrait néanmoins les conditions essentielles «[...] à une émergence d'une ou plusieurs sciences juridiques au Canada» (p. 525). Par ses propos, monsieur Laperrière ne semble pas partager l'opinion exprimée aux pages suivantes par Pierrot Péladeau qui postule que: «[...] La recherche juridique ne peut devenir scientifique qu'en tentant de se dégager du discours idéologique par l'explication à la fois de son point de vue et de celui du droit étudié» (p. 528). Ce dernier article entend dévoiler les atteintes réelles à la liberté que pourraient commettre ceux qui disposent de banques de données informatisées. Cet auteur montre bien que le juriste seul ne pourrait aborder ces questions et qu'il doit s'agir d'une intervention scientifique et d'une pratique multidisciplinaire. Guy Rocher dresse un bilan de la sociologie du droit au Québec. On y relate certaines études sociologiques et analyses de la genèse et des facteurs qui incitèrent le législateur à intervenir en plusieurs champs: l'éducation, la santé, les corporations professionnelles, etc. On souligne également l'usage du recours judiciaire (le one-man lobby) et l'apport des tribunaux à la production juridique. Puis, l'auteur nous rappelle des études faites sur la personne même des juges, le fonctionnement de l'appareil judiciaire, le rôle des avocats et finalement, les écarts entre la lettre de la loi, son application et ses effets. En somme, l'auteur souligne à quel point une étude méthodique du droit et de ses effets permettrait de découvrir sa fonction réelle dans la société. Un dernier article traite «des pratiques alternatives en droit» rédigé par Katherine Lippel. Il s'agirait de cette pratique dont la clientèle est constituée de toutes ces personnes ou groupes «[...] qui ne détiennent pas le pouvoir» c'est-à-dire les autochtones, les détenus, les immigrants, les accidentés, les chômeurs, 
les assistés sociaux, les handicapés, etc. Katherine Lippel souligne qu'il y aurait pénurie d'avocats pour une telle pratique alors que «[...] le Barreau du Québec s'inquiète de la saturation du marché, il reste des milliers de clients non desservis par les avocats» (p. 598). Cette pratique comprendrait l'utilisation politique du droit et de multiples manières: «[...] Le rôle du juriste, c'est de garantir ce droit de parole; le rôle du militant et de la militante est de s'en servir» (p. 599) ou encore, «[...] de fournir les outils juridiques permettant la repolitisation d'une question politique judiciarisée» et même de revendiquer le «droit au chauffage». On rapporte par la suite, à titre d'illustration, de nombreuses affaires où l'on utilisa ces approches. Parlant sans doute d'expérience, l'auteure nous fait part de la difficulté de vivre une telle pratique: «On a beau faire les meilleures analyses sur le rôle du droit et de la justice, il faut quand même plusieurs années pour bien intégrer le principe qu'on a gagné en perdant un procès. [...] On a beau savoir que l'échec judiciaire peut mener à la victoire politique, il est quand même difficile d'avoir constamment l'impression de se cogner la tête contre un mur» (p. 617).

En raison de son caractère propre et de la lecture particulière du droit que nous proposent ces trente auteurs, il n'y a pas que les étudiants des facultés qui auraient intérêt à lire cet ouvrage. Avocats certes, mais aussi politicologues, sociologues et historiens pourraient y puiser un enseignement stimulant et permettant même certaines remises en cause. En attendant, espérons que cette jeune équipe de juristes publieront davantage et fourniront quelques pistes ou éléments de réponse aux très nombreuses questions qu'ils ont eux-mêmes soulevées en 1987.

Fernand MORIN

Université Laval

Flexibilité and Labour Markets in Canada and the United States (Research Series 94) by Gilles Laflamme, Gregor Murray, Jacques Bélanger and Gilles Ferland (eds.), Geneva, International Institute for Labour Studies, 1989, 317 pp., ISBN 92-9014-462-9

Economic flexibility, including how employers allocate labour, has been an important issue over the past decade. Catalyzed by the relative decline of North American industry over the past two decades, labour flexibility involves lifting or loosening up restrictions placed upon employers in their allocation of labour. And though many would have us believe that this is a relatively new issue, labour flexibility has been around for decades in such forms as contracting out, lay offs, job enlargement and job enrichment schemes. Though it may be the case of old wine in new bottles, economic conditions have brought about a renewed debate on labour flexibility, on the level of the national and international economies as well as within the enterprise.

Unlike earlier critiques from the business community or classical economists which were aimed at specific state and trade union «imposed» restrictions on the allocation of labour, the entire "post-war accord», described by some as «Fordism», has come under increasing criticism for being inflexible. «Fordism» has been described as an historically specific form of capitalism which saw economic prosperity in post World War II North America founded upon such production techniques as the assembly line and such institutional arrangements as industry-wide pattern bargaining and the welfare state. The traditional assembly line has long been for dehumanizing work and for being locked into an inflexible strategy of standardized mass production. Advocates of shop floor «flexibility" recommend modifying or dismantling of the Taylorist assembly line, so as to increase product quality, make industry more responsiveness to consumer demands and to create more fulfilling work. 